This is the post peer-review accepted manuscript of:

A. Guerra, F. Guidi, A. Clemente, R. D'Errico, L. Dussopt and D. Dardari, "Millimeter-Wave Backscattering Measurements with Transmitarrays for Personal Radar Applications," 2015 IEEE Globecom Workshops (GC Wkshps), San Diego, CA, 2015, pp. 1-6. https://doi.org/10.1109/GLOCOMW.2015.7414160

The published version is available online at:

http://ieeexplore.ieee.org/stamp/stamp.jsp?tp=\&arnumber=7414160\&isnumber=7413928

(C) 2015 IEEE. Personal use of this material is permitted. Permission from IEEE must be obtained for all other uses, in any current or future media, including reprinting/republishing this material for advertising or promotional purposes, creating new collective works, for resale or redistribution to servers or lists, or reuse of any copyrighted component of this work in other works 


\title{
Millimeter-wave Backscattering Measurements with Transmitarrays for Personal Radar Applications
}

\author{
Anna Guerra*, Francesco Guidi ${ }^{\dagger \ddagger}$, Antonio Clemente ${ }^{\dagger \ddagger}$, Raffaele D’Errico ${ }^{\dagger \ddagger}$, Laurent Dussopt ${ }^{\dagger \ddagger}$, and Davide Dardari* \\ ${ }^{*}$ DEI, University of Bologna, via Venezia 52, I-47521 Cesena (FC), Italy \\ \{anna.guerra3, davide.dardari\}@unibo.it \\ †Univ. Grenoble-Alpes, 38000 Grenoble, France \\ $\ddagger$ CEA, LETI, MINATEC Campus, 38054 Grenoble, France \\ \{francesco.guidi, antonio.clemente, raffaele.derrico, laurent.dussopt\}@cea.fr
}

\begin{abstract}
The concept of personal radar has recently emerged as an interesting solution for next 5G applications. In fact the high portability of massive antenna arrays at millimeterwaves enables the integration of a radar system in pocket-size devices (i.e. tablets or smartphones) and enhances the possibility to map the surrounding environment by guaranteeing accurate localization together with high-speed communication capabilities. In this paper we investigate for the first time the capability of such personal radar solution using real measured data collected at millimeter-waves as input for the mapping algorithm.
\end{abstract}

Index Terms-Millimeter-wave propagation measurements, massive antenna arrays, indoor mapping, personal radar.

\section{INTRODUCTION}

In the new era of device-to-device (D2D) communications, claimed by the fifth generation $(5 \mathrm{G})$ wireless mobile networks, people and objects will share their information in real time, as data transfer could be enabled at an unprecedented scale. At the moment, 5G is still at its infancy, as its practical adoption is expected in the next decade [1], with a consequent explosion of research studies and projects for its assessment. To put forth on the need to achieve high data rates and D2D communication, massive arrays and millimeter-wave $(\mathrm{mmW})$ technology have become a matter of investigation [2], [3]. It is expected that their joint adoption will play a key role in future $5 \mathrm{G}$ communications scenarios [4]. Indeed, the reduced wavelength at $\mathrm{mmW}$ paves the way for packing a large number of antenna elements into a small area and for adopting massive arrays systems at base station (BS) or access points (APs) as well as at mobile end [2], [5].

In this context, transmitarrays (TAs) are a recent cuttingedge antenna array technology. Thanks to their spatial feeding techniques, TAs are extremely attractive compared to traditional phased arrays that suffer from large insertion losses due to their beamforming network and do not experience any feed blockage effects if compared to reflector and reflectarray antennas [6]-[8]. Studies concerning the adoption of $\mathrm{mmW}$ TAs can be found in [6]. Note that such reduced dimensions open new interesting scenarios for the integration of arrays on smartphones and for the development of new applications.

In this perspective, the concept of a smart personal radar has been recently proposed in [9]-[11] as well as the adoption of TAs for this kind of application [12]. The personal

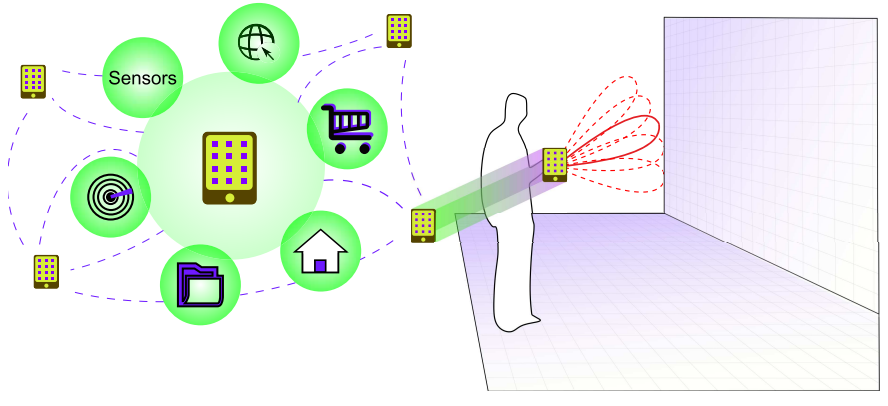

Fig. 1. Smartphone centric solution for next crowd-sensing applications (left) and personal radar concept (right).

radar is based on the idea of a massive array operating at $\mathrm{mmW}$ frequencies able to electronically scan the surrounding environment and to reconstruct a map of it (see Fig. 1). As a consequence, a high-definition distance estimate (ranging) and very narrow steering beams (angle resolution) are essential requirements to achieve a high level of mapping reconstruction. Differently from previous works in the field of mapping and localization [13], such needs are met by the combination of the $\mathrm{mmW}$ technology and massive arrays.

Moreover, the possibility to share data within the incoming crowd-sensing technology [14] opens unprecedented scenarios where continuously up-to-date maps could be obtained with zero-efforts exploiting not participatory users. In fact, to avoid invasive and costly ad-hoc systems to support people (e.g., visually impaired people) and perform self-localization, the adoption of personal radars in next generation smartphones represents an appealing idea [15].

In this paper we aim at characterizing real mapping performance by exploiting, for the first time, measurements collected in the V-band in a typical office environment. Stimulated by the preliminary simulation results presented in [9], [12], we have conducted a $\mathrm{mmW}$ measurements campaign with the purpose of assessing the actual system performance and the feasibility of the personal radar idea. We first describe the measurements campaign and then we include the collected data in the mapping algorithm in order to reconstruct the environment and to test the feasibility of the proposed ap- 


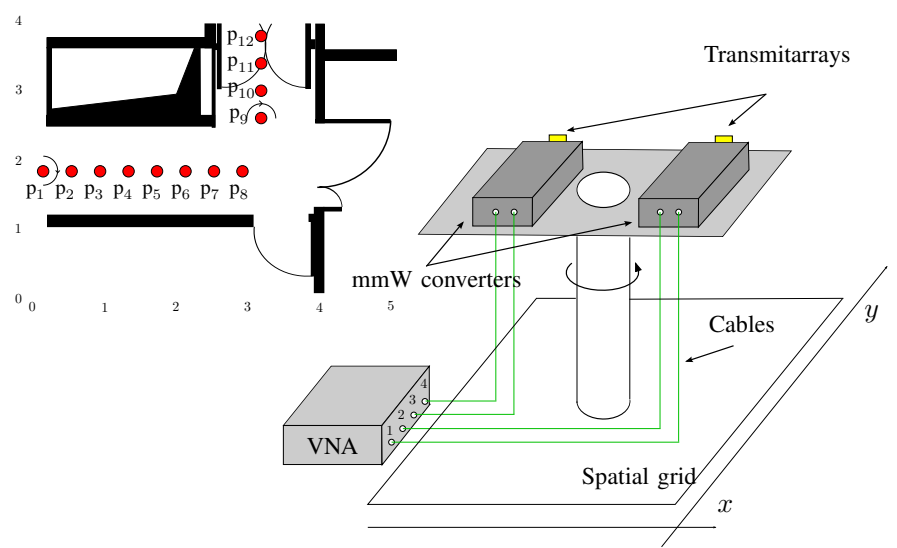

Fig. 2. Plan of the indoor corridor environment (top-left) and measurement set-up (right).

proach. Measurement mapping results are finally compared with simulation ones.

The rest of the paper is organized as follows. Sec. II describes the measurement set-up and the considered TAs. Sec. III presents the mapping algorithm and finally, Sec. IV describes the case study and discuss the obtained measurement and simulations results. Sec. V concludes the work.

\section{Millimeter-WAVE INDOOR MEASUREMENT CAMPAIGN}

\section{A. Measurements Set-Up}

In order to validate the personal radar concept, a measurements campaign has been conducted at CEA-Grenoble indoor premises (the map of the building floor is reported in Fig. 2(top-left)).

In particular, we exploited the measurement set-up represented in Fig. 2-(right) which consists of:

- A 4-ports Vector Network Analyzer (VNA) operating in the frequency range $10 \mathrm{MHz}-24 \mathrm{GHz}$;

- $2 \mathrm{mmW}$ converters operating in the frequency range $50 \mathrm{GHz}-75 \mathrm{GHz}$;

- 2 Linearly polarized TAs (size $20 \times 20,1$ bit, $F / D=0.5$ ) [16];

- $X$-Y-Azimuth positioner.

A bistatic configuration has been considered with the TAs spaced apart of $0.16 \mathrm{~m}$ in order to mitigate the antenna coupling and to separate the transmitting and receiving channels. As the objective application is to facilitate the navigation inside buildings (as for example blind-aid applications), the corridor has appeared as a natural choice. Specifically, as we can see from Fig. 2-(top-left) where the office plan is reported, measurements have been collected in 12 different positions spaced of $0.405 \mathrm{~m}^{1}$ in the frequency range between $55-70 \mathrm{GHz}$ with a step of $5 \mathrm{MHz}$. For each measurement position, the $X$-Y-Azimuth positioner rotates the radar in the semi-plane from $-90^{\circ}$ to $90^{\circ}$ with a step of $5^{\circ}$ in accordance

\footnotetext{
${ }^{1}$ This step has been chosen in accordance to the value of the fully automated movement along the $y$-axis achievable with the positioner.
}
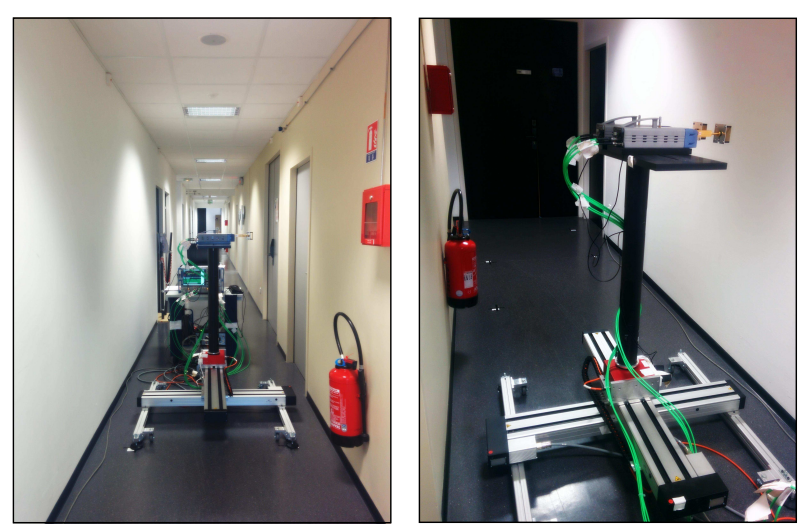

Fig. 3. Photos of measurements campaign in the corridor.

to the TA half power beamwidth (HPBW) to emulate the beamforming operation. Such mechanical steering reproduces an electronical steering since the adopted TAs are not reconfigurable. Technological solutions to perform electronically beamsteering at V-band can be found in [17] where a multiplesource system is proposed. Fig. 3 shows two photos taken during the measurements campaign.

\section{B. Transmitarray antenna}

The TAs used for measurements are fully described in [16] and here we report the most important information useful for the reader to appreciate how TAs radiation properties impact the mapping performance. As can be seen in Fig. 4, the TA is composed of a focal source illuminating a planar array whose building blocks are called unit-cells. In the considered TA structure, $20 \times 20$ unit-cells are adopted each with size of $2.5 \times 2.5 \mathrm{~mm}^{2}$ (i.e. the spacing between antennas is set to $\lambda_{0} / 2=2.5 \mathrm{~mm}$ where $\lambda_{0}$ is the wavelength at $\left.f_{0}=60 \mathrm{GHz}\right)$. Each unit-cell is composed of two patch antennas (receiver and transmission one) printed on two identical substrates separated by a ground plane and connected by a metallized via hole. The transmission phase can be controlled by rotating the patch on the transmission side. As previously introduced, this TA is non-reconfigurable because the patches rotation is fixed once the array is realized. The considered TA presents 1-bit of phase compensation corresponding to two possible phase values $\left(0^{\circ}\right.$ and $\left.180^{\circ}\right)$. Finally, the focal source is a linearlypolarized pyramidal horn antenna with a gain equal to $10.2 \mathrm{dBi}$ at $60 \mathrm{GHz}$ and a full HPBW of $72^{\circ} \times 57^{\circ}$.

Given this array structure, in [16] simulations and measurements results report a maximum gain of $23.3 \mathrm{dBi}$, a directivity, spill-over loss and power efficiency equal to $26 \mathrm{dBi}, 2.24 \mathrm{~dB}$, and $53.6 \%$, respectively.

In Fig. 5-(top) we report the maximum gain of the TA as a function of the frequency range used for measurements. The dashed lines correspond to the gain at $3 \mathrm{~dB}$ and intercept the continuous curve in correspondence of two frequency values, $f_{L}=55 \mathrm{GHz}$ and $f_{H}=69 \mathrm{GHz}$, which define the extremes of the $3 \mathrm{~dB}$ bandwidth. In Fig. 5-(bottom) the phase distribution and the realized gain are shown. Note that we have reported the 


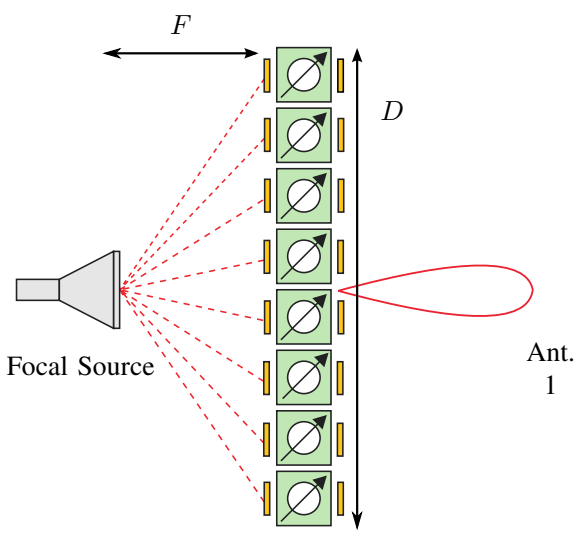

(a) $\underline{\text { Unit-cell }}$

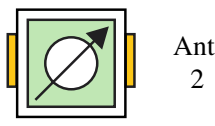

Phase shifter

(b)
Fig. 4. TA structure: (a) TA configuration and (b) unit-cell.

realized gain for $f_{L}, f_{H}$ and for the frequency $f_{\mathrm{c}}=60 \mathrm{GHz}$ at which the TA has been optimized.

\section{Measurement Results}

For each position and each TA steering direction, the $S$ matrix was measured in the frequency domain from 55 to $70 \mathrm{GHz}$. In particular, the measured $\mathrm{S}_{21}(f)$ has been translated in time-domain through the inverse Fourier transform, and time gating was performed in order to suppress the coupling between the two arrays.

Successively, the round-trip channel impulse response (CIR), representative of the two-hop backscattering link, has been convoluted with a root raised cosine (RRC) pulse, with the effective radiated isotropic power (EIRP) compliant with the Federal Communications Commission (FCC).

In Figs. 6, 7 the CIR $s_{21}(\tau)$, the received signal and the energy profile collected from position $\mathrm{p}_{6}$ for two different steering angles $\left(\theta_{b}=0^{\circ}\right.$ and $\theta_{b}=-45^{\circ}$, respectively) are shown. The energy profile has been obtained by considering $N_{\text {bin }}$ time bins of duration $T_{\mathrm{ED}}$. A signal bandwidth of $W=2 \mathrm{GHz}\left(T_{\mathrm{ED}}=0.5 \mathrm{~ns}\right)$, a time frame $T_{\mathrm{f}}=100 \mathrm{~ns}$ $\left(N_{\text {bin }}=200\right)$ and $N_{\mathrm{p}}=100$ transmitted pulses have been taken into account. As we can see, when the steering angle is fixed to $\theta_{b}=0^{\circ}$, the received signal presents a peak in correspondence to $13 \mathrm{~ns}$ approximately. This refers to the time-of-arrival (TOA) related to the reflection of the wall in front of the radar at the end of the corridor. Thanks to the high directivity and gain of the adopted TAs, all the other reflections are filtered out or strongly mitigated. Contrarily, when $\theta_{b}=-45^{\circ}$, a first peak in the received signal can be detected at $5 \mathrm{~ns}$ related to the left-hand wall side while the highest peak is at approximately $18 \mathrm{~ns}$ probably corresponding to the wall in the direction of the main beam.

\section{MAPPING ALGORITHM}

As previously anticipated, the goal of this paper is to provide the feasibility of the mapping approach by exploiting measurement data, and compare them with simulations. Thus, the grid-based state-space model for probabilistic mapping
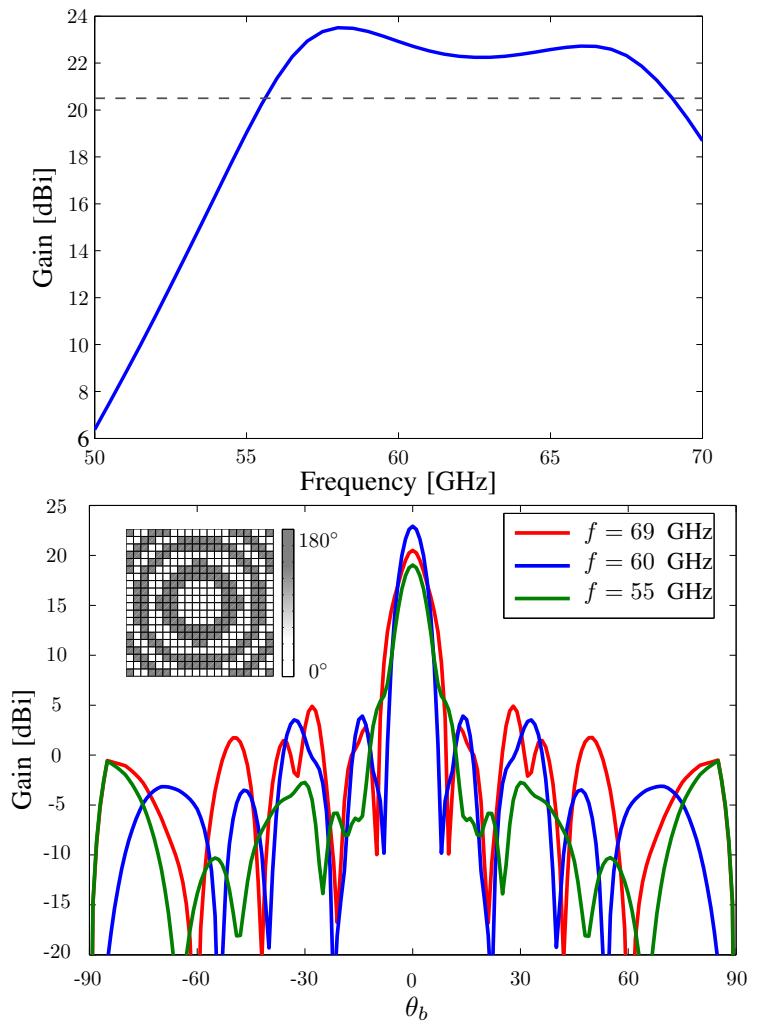

Fig. 5. Computed maximum gain of the TA used for measurements for $\theta_{b}=0^{\circ}$ as a function of frequencies (top), phase distribution and realized gain for $f_{L}, f_{H}$ and $f_{\mathrm{C}}$ (bottom) [6].

we proposed in [11] has been considered, by including alternatively both simulated and real measured data. Since we suppose that the personal radar trajectory and orientation are obtained using a dedicated positioning infrastructure, here we focus only on mapping and not on simultaneous localization and mapping (SLAM).

As reported in Fig. 1, the personal radar explores an unknown environment by performing a beamsteering operation during which its main beam is oriented in different spatial directions. For each position and each steering direction, the radar emits a train of $N_{\mathrm{p}}$ pulses and then it collects the respective backscattered response. Energies are evaluated by subdividing the time frame into $N_{\text {bin }}=\left\lfloor T_{\mathrm{f}} / T_{\mathrm{ED}}\right\rfloor$ time slots (bins) of duration $T_{\mathrm{ED}} \approx 1 / W$, which are successively accumulated for $N_{\mathrm{p}}$ times [11].

The environment has been discretized in $N_{\mathrm{L}}=X_{\text {grid }} Y_{\text {grid }}$ cells, where each cell is associated with a radar cross section (RCS) value to be inferred by the estimation process. The cell size is set in accordance to the minimum footprint area, as detailed in Sec. IV.

The state vector of the system here considered is given by

$$
\mathbf{x}(k)=\mathbf{m}(k)=\left[m_{1}(k), \ldots, m_{i}(k), \ldots, m_{N_{\mathrm{L}}}(k)\right]^{\mathrm{T}}
$$

where $k$ is the discrete time instant and $m_{i}(k)$ indicates the root radar cross section (RRCS) (with sign) of the $i$ th cell of the grid, where the frequency dependency has been 


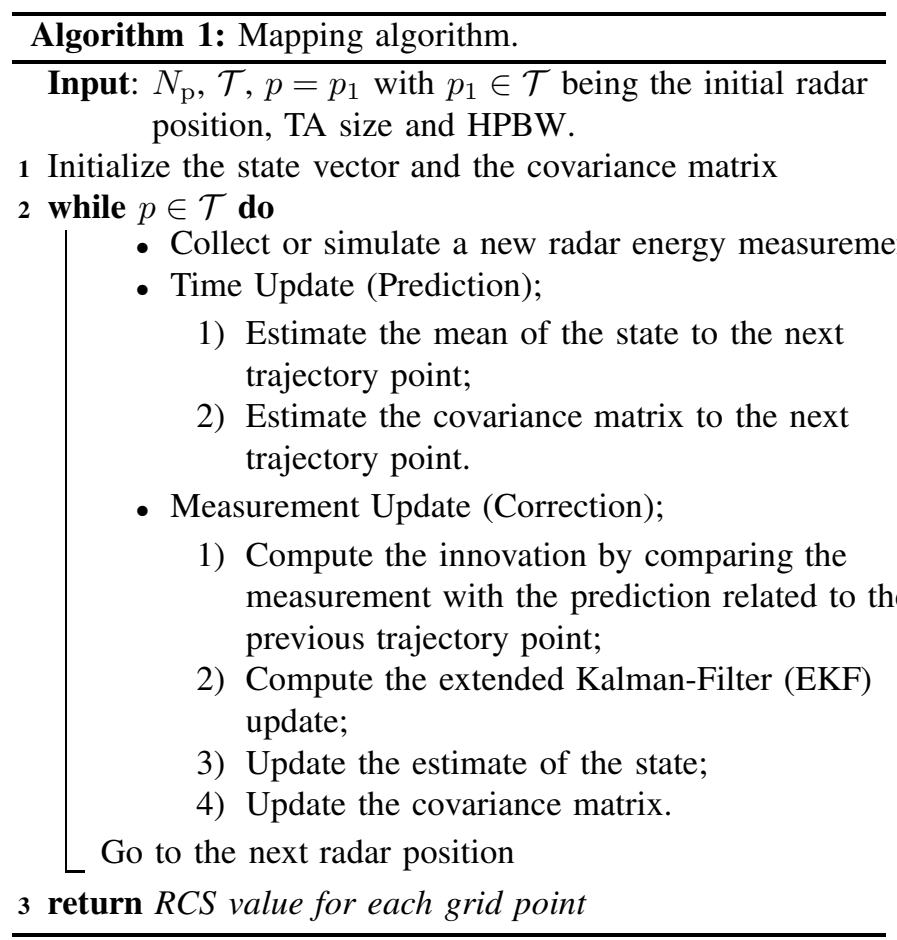

neglected. Moreover, since we assume stationary environment, the transition model is not introduced.

Contrarily to conventional radar approaches where environment mapping is preceded by a detection phase [13], [18], in our model all the available energy measurements are included in the observation model. Specifically, we define $\mathbf{e}(k)$ the vector containing the accumulated measured energy at the output of the receiver at time $k, \mathcal{T}$ the set of points inside the room which belongs to the radar trajectory and we indicate with $\mathbf{z}(k)$ the corresponding Gaussian observation model fully described in [9].

The map estimation is performed by adopting the EKF method to efficiently evaluate the posterior distribution $p(\mathbf{x}(k) \mid \mathbf{e}(1: k))$ of $\mathbf{x}(k)$ given the set of measurements $\mathbf{e}(1: k)=\{\mathbf{e}(1), \mathbf{e}(2), \ldots, \mathbf{e}(k)\}$ collected by the TA-radar until time instant $k$, from which a maximum a posteriori estimate of the state $\mathbf{x}(k)$ is derived.

More details on how the mapping algorithm is performed are reported in Algorithm 1 and in [9], [19].

\section{CASE STUdY}

We now describe the mapping results obtained with the proposed model by including both simulated and measured data.

\section{A. Scenario and System Parameters}

The case study here described refers to the corridor environment described in Sec. II-A whose layout is juxtaposed to the estimated RCS maps in the results. As previously mentioned, we adopt a grid-based approach where the environment is discretized in cells having area $0.1 \times 0.1 \mathrm{~m}^{2}$ each according to the TA radiation patterns. We consider the radar equipped with
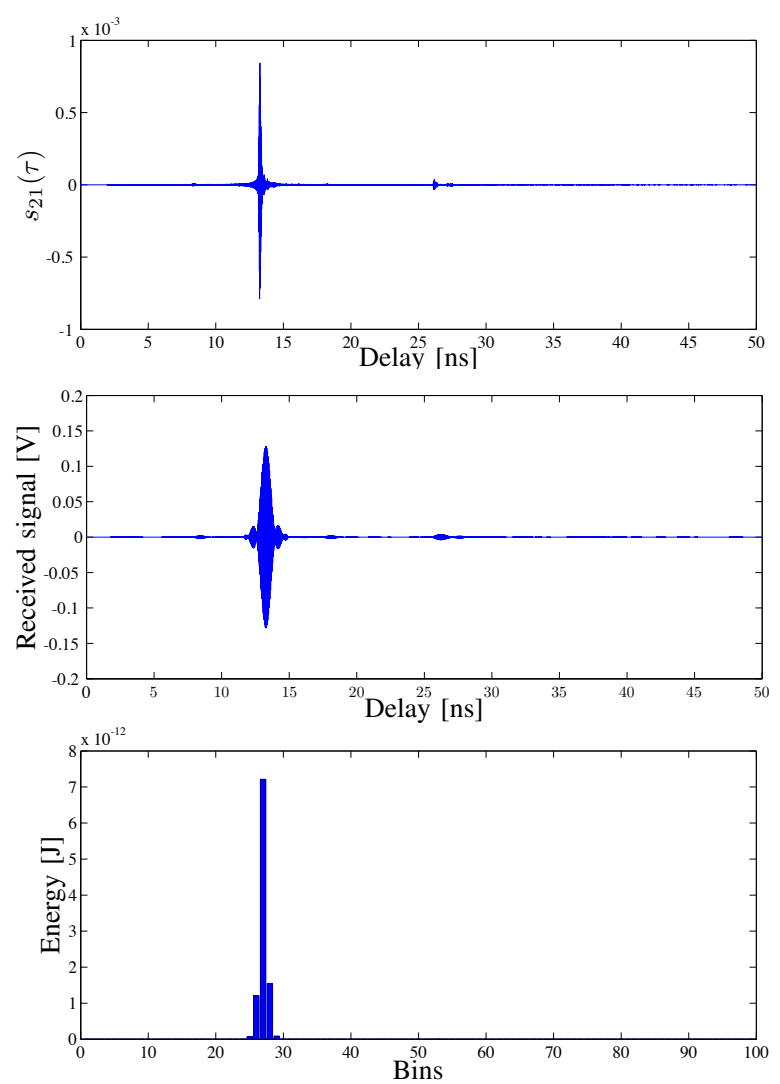

Fig. 6. Example of CIR (top), received signal (middle) and of the correspondent accumulated energy profile (bottom) taken from position $p_{6}$ in the corridor, for a steering direction of $\theta_{b}=0^{\circ}, W=2 \mathrm{GHz}$ and $N_{p}=100$.

the TA described in Sec. II-B, exploring the surrounding area and collecting energy measurements by performing a scanning process over an angular range of $\left(-90^{\circ}, 90^{\circ}\right)$ with a step of $5^{\circ}$ as described in previous sections. The TA-radar moves along a determined and known path, denoted by the red circles in Fig. 4, with a spatial step of $0.405 \mathrm{~m}$. The orientation of the radar is always in the sense of the movement. In this scenario the TA-radar position and orientation are known; however, in a more realistic scenario errors in the position/orientation estimation have to be accounted for. In [9] the effects of such errors have been considered in the simulation results to analyse how they could impact in the map reconstruction.

We compare the TA mapping capabilities in terms of the signal bandwidth and by taking into account the quantization effect introduced by the 1-bit phase shift. A noise figure of $N_{\mathrm{F}}=4 \mathrm{~dB}, T_{0}=290 \mathrm{~K}$ and a signal bandwidth of $W=$ $1-2-3 \mathrm{GHz}$ (with $T_{\mathrm{ED}}=1 / W$ ) are considered. RRC pulses centered at frequency $f_{\mathrm{c}}=60 \mathrm{GHz}$, roll-off factor $\alpha=0.6$, and pulse width parameter $t_{\mathrm{p}}=(1+\alpha) / W$ have been adopted. The time frame has been set to $100 \mathrm{~ns}$ and the number of transmitted pulses to $N_{\mathrm{p}}=100$.

Results are obtained considering an EIRP of $30 \mathrm{dBm}$ in accordance to Federal Communications Commission (FCC) mask at $60 \mathrm{GHz}$ [20]. 

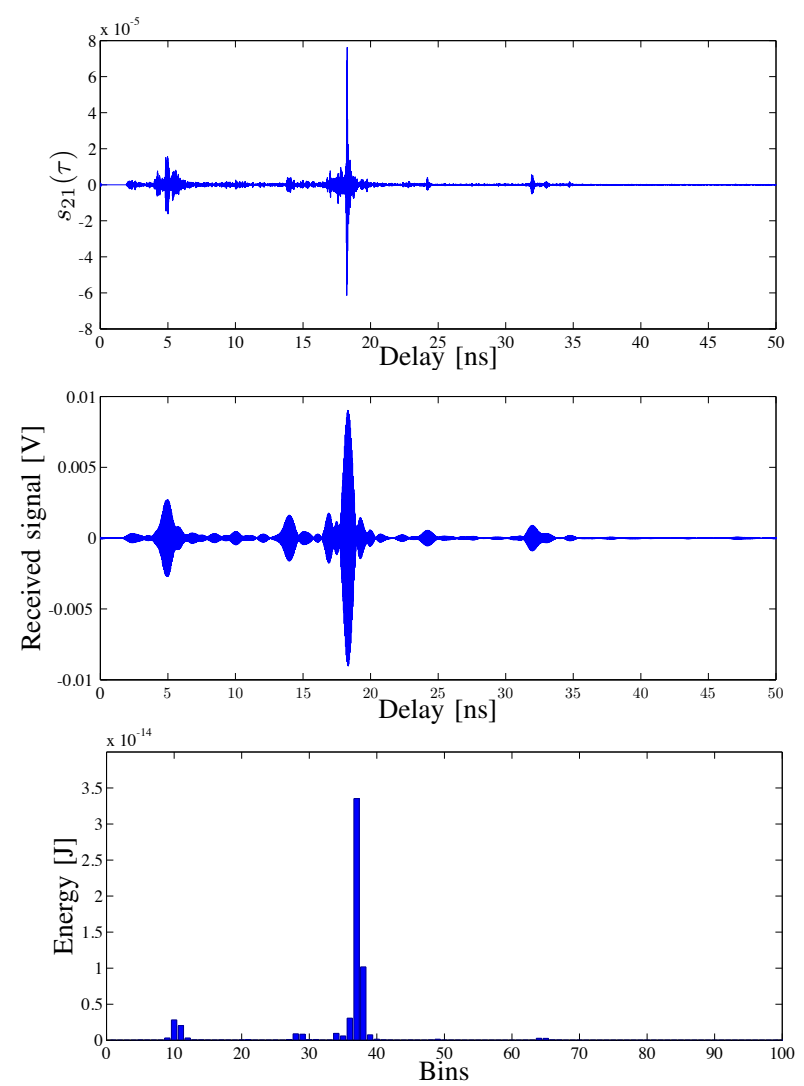

Fig. 7. Example of CIR (top), received signal (middle) and of the correspondent accumulated energy profile (bottom) taken from position $p_{6}$ in the corridor, for a steering direction of $\theta_{b}=-45^{\circ}, W=2 \mathrm{GHz}$ and $N_{p}=100$.

\section{B. Mapping Results with Measured Energy Vectors}

In this case, the measurement vector $\mathbf{e}(k)$ is given by data collected in Sec. II, where the energy profiles are directly included.

In Fig. 8 the mapping results exploiting the accumulated energy measurements are reported as a function of the signal bandwidth. Specifically, from the left to the right the RCS estimated map obtained with $W=1 \mathrm{GHz}$ (i.e. $T_{\mathrm{ED}}=1 \mathrm{~ns}$ ), $W=2 \mathrm{GHz}$ (i.e. $T_{\mathrm{ED}}=0.5 \mathrm{~ns}$ ) and $W=3 \mathrm{GHz}$ (i.e. $T_{\mathrm{ED}}=0.34 \mathrm{~ns}$ ) are shown. As expected, when increasing the bandwidth the mapping performance improves due to the increased temporal resolution.

Results obtained with $W=3 \mathrm{GHz}$ are quite satisfactory as there is a nice match between the real maps and those estimated.

\section{Mapping Results with Simulated Energy Vectors}

We then simulate the measurements step taking into account the RCS scattering model described in [21] with a wall made of aerate concrete (i.e. relative permittivity equal to $\epsilon_{r}=2.26$ and its loss tangent to 0.0491) and by exploiting the mapping model proposed in [19]. In this scenario, we have considered the radar moving with a speed of $1 \mathrm{~m} / \mathrm{s}$ and taking measurements every $0.4 \mathrm{~s}$. The radiation characteristics of the
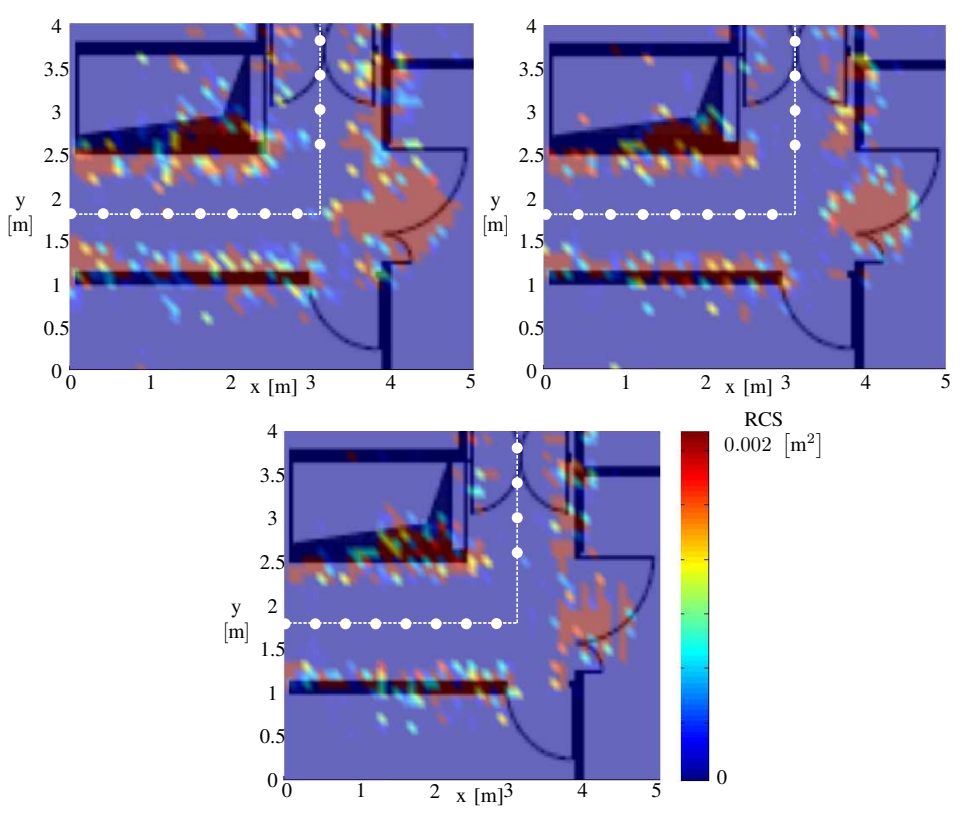

Fig. 8. Mapping results using 1-bit $20 \times 20$ transmitarrays, $W=1-2-$ $3 \mathrm{GHz}$ (top-left, top-right and bottom, respectively), $N_{\mathrm{p}}=100$ and exploiting real measured data.

1-bit $20 \times 20$ TAs used for measurements have been included in the simulator. As before, in Fig. 9 the simulation results are reported as a function of the signal bandwidth. Discrepancies between the previous results are due to different phenomena. First of all in the simulations a simple scattering model has been assumed. As a consequence, when considering collected measurements as an input for the mapping simulator a mismatch between the model and the real scattering behaviour can cause a performance degradation. Secondly, in simulations we have assumed a free space propagation condition for each interrogated cell supposing that the multipath effects is implicitly taken into account when including all the array pattern information in the grid-based approach. Obviously a more realistic channel model might help to fill the gap between simulations and measurements. Finally, in the simulation results the far-field assumption has been made. Thanks to this hypothesis, it is possible to exploit the far-field TA radiation pattern both in the prediction and the measurement phases when evaluating the mapping performance. Contrarily, in the real scenario where the measurements have been collected in a corridor (1.6 m width) the far-field condition is not always satisfied due to the high TA directivity with the consequence that the expected TA main beam could be not perfectly formed. This fact could cause a mismatch between the prediction and the measurement step of the mapping algorithm. Future works will be addressed in improving the mapping algorithm by properly accounting for such phenomena.

\section{CONCLUSIONS}

In this paper we present an indoor backscattering measurement campaign at millimeter-wave for personal radar mapping applications. Two 1-bit $20 \times 20$ linearly polarized TAs have 

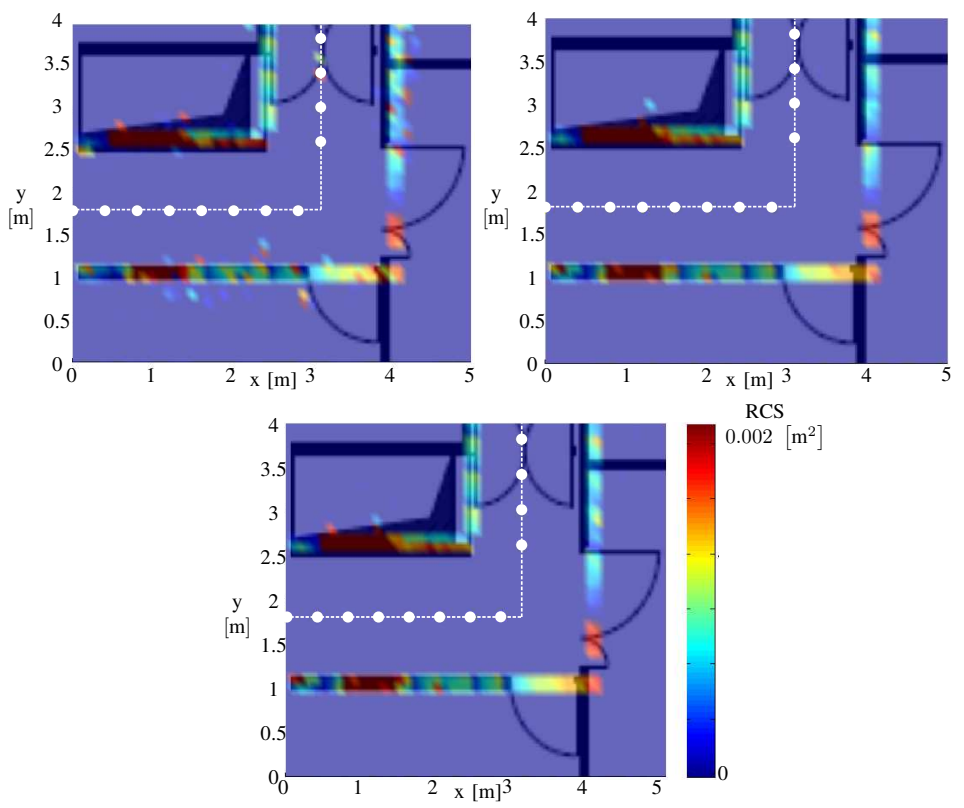

Fig. 9. Mapping results using 1-bit $20 \times 20$ transmitarrays, $W=1-2-$ $3 \mathrm{GHz}$ (top-left, top-right and bottom, respectively), $N_{\mathrm{p}}=100$ and exploiting simulated data.

been used in a bistatic radar configuration to scan a real office environment. The data collected have been exploited for map reconstruction using a grid-based Bayesian statespace approach. Results have demonstrated the feasibility of the $\mathrm{mmW}$ personal radar, previously proved only through simulations, and that a good quality of map reconstruction can be achieved even when a limited set of phase values, i.e. only two in our case, are available.

\section{ACKNOWLEDGMENT}

This work has been supported by the Italian Ministerial PRIN project GRETA (Grant 2010WHY5PR), H2020 project XCycle and in part by the H2020-EU.1.3.2 IF-EF Marie-Curie project MAPS (Grant 659067).

\section{REFERENCES}

[1] http://www.huawei.com/5gwhitepaper/.

[2] F. Rusek et al., "Scaling up MIMO: Opportunities and challenges with very large arrays," IEEE Signal Processing Mag., vol. 30, no. 1, pp. 40-60, 2013.

[3] T. Rappaport et al., "Millimeter wave wireless communications." Prentice Hall, 2014.

[4] F. Boccardi et al., "Five disruptive technology directions for 5G," IEEE Commun. Mag., vol. 52, no. 2, pp. 74-80, February 2014.

[5] W. Hong et al., "Study and prototyping of practically large-scale mmwave antenna systems for 5G cellular devices," IEEE Commun. Mag., vol. 52, no. 9, pp. 63-69, September 2014.

[6] H. Kaouach et al., "Wideband low-loss linear and circular polarization transmit-arrays in V-band," IEEE Trans. Antennas Propag., vol. 59, no. 7, pp. 2513-2523, July 2011.

[7] A. Clemente et al., "Multiple feed transmit-array antennas with reduced focal distance," in Proc. 42nd European Microwave Conf. (EuMC), Oct 2012, pp. 826-829.

[8] L. Di Palma et al., "Circularly polarized transmit-array with sequentially rotated elements in Ka band," in Proc. 8th European Conf. on Antennas and Propag. (EuCAP), April 2014, pp. 1418-1422.
[9] F. Guidi, A. Guerra, and D. Dardari, "Personal mobile radars with millimeter-wave massive arrays for indoor mapping," IEEE Trans. on Mobile Comp., vol. 14, no. 99, 2015.

[10] - "Millimeter-wave massive arrays for indoor SLAM," in Proc. IEEE Int. Conf. on Commun. (ICC), June 2014, pp. 114-120.

[11] A. Guerra, F. Guidi, and D. Dardari, "Millimeter-wave personal radars for 3D environment mapping," in Proc. IEEE Asilomar Conf. on Signals, Systems, and Computers, Pacific Grove, USA, Nov. 2014.

[12] A. Guerra et al., "Application of transmitarray antennas for indoor mapping at millimeter-waves," in Proc. IEEE European Conf. on Networks and Commun. (EUCNC), 2015.

[13] E. Jose and M. Adams, "An augmented state SLAM formulation for multiple line-of-sight features with millimetre wave radar," in Proc. IEEE/RSJ Int. Conf. Intelligent Robots and Syst., Aug. 2005, pp. 30873092.

[14] C. Wu, Z. Yang, and Y. Liu, "Smartphones based crowdsourcing for indoor localization," IEEE Trans. Mobile Comp., vol. PP, no. 99, pp. 1-1, 2014.

[15] D. Dardari et al., "A combined GP-state space method for efficient crowd mapping," in Proc. IEEE Int. Conf. on Commun. (ICC), June 2015.

[16] H. Kaouach et al., "Wideband low-loss linear and circular polarization transmit-arrays in v-band," IEEE Trans. on Antennas and Propag., vol. 59, no. 7, pp. 2513-2523, July 2011.

[17] J. A. Zevallos Luna and L. Dussopt, "A V-band switched-beam transmitarray antenna," International Journal of Microwave and Wireless Technologies, vol. 6, pp. 51-56, 22014.

[18] E. Jose et al., "Predicting Millimeter Wave Radar Spectra for Autonomous Navigation," IEEE Sensors J., vol. 10, no. 5, pp. 960-971, May 2010.

[19] A. Guerra, F. Guidi, and D. Dardari, "Position and orientation error bound for wideband massive antenna arrays," in Proc. IEEE Int. Conf. on Commun. (ICC), ANLN Workshop, 2015.

[20] "Revision of Part 15 of the Commissions Rules Regarding Operation in the 57-64 GHz Band," Federal Communications Commission (FCC), Aug. 2013.

[21] L. Correia and P. Frances, "Estimation of materials characteristics from power measurements at $60 \mathrm{GHz}$," in Proc. IEEE 5th Int. Symp. on Personal, Indoor and Mobile Radio Commun. (PIRMC), 1994, pp. 510513 vol.2. 